\title{
Antibacterial and Antibiofilm Effect of Cell-Free Supernatant of Lactobacillus brevis KCCM 202399 Isolated from Korean Fermented Food against Streptococcus mutans KCTC 5458
}

\author{
Jong Ha Kim, Hye Ji Jang, Na-Kyoung Lee, and Hyun-Dong Paik* \\ Department of Food Science and Biotechnology of Animal Resource Konkuk University, Seoul 05029, Republic of \\ Korea
}

\begin{abstract}
This study aims to determine the antibiofilm effect of cell-free supernatant (CFS) of Lactobacillus brevis strains against Streptococcus mutans strains. To study the antibiofilm mechanism against S. mutans strains, antibacterial effects, cell surface properties (auto-aggregation and cell surface hydrophobicity), exopolysaccharide (EPS) production, and morphological changes were examined. The antibiofilm effect of $L$. brevis KCCM 202399 CFS as morphological changes were evaluated by scanning electron microscopy (SEM) and confocal laser scanning microscopy (CLSM), compared with the control treatment. Among the L. brevis strains, L. brevis KCCM 202399 showed the highest antibiofilm effect on S. mutans KCTC 5458. The antibacterial effect of L. brevis KCCM 202399 against S. mutans KCTC 5458 was investigated using the deferred method $(16.00 \mathrm{~mm})$. The minimum inhibitory concentration of $L$. brevis KCCM 202399 against S. mutans KCTC 5458 was $25.00 \%$. Compared with the control treatment, L. brevis KCCM 202399 CFS inhibited the bacterial adhesion of S. mutans KCTC 5458 by decreasing auto-aggregation, cell surface hydrophobicity, and EPS production (45.91\%, 40.51\%, and 67.44\%, respectively). L. brevis KCCM 202399 CFS inhibited and eradicated the S. mutans KCTC 5458 biofilm. Therefore, these results suggest that $L$. brevis KCCM 202399 CFS may be used to develop oral health in the probiotic industry.
\end{abstract}

Keywords: Probiotics, antibacterial effect, antibiofilm effect, Streptococcus mutans

Received: September 24, 2021 Accepted: October 15, 2021

First published online: October 17, 2021

*Corresponding author Phone: +82-2-2049-6011 E-mail: hdpaik@konkuk.ac.kr

pISSN 1017-7825 elSSN 1738-8872

Copyright $\odot 2022$ by the authors. Licensee KMB. This article is an open access article distributed under the terms and conditions of the Creative Commons Attribution (CC BY) license.

\section{Introduction}

Streptococcus mutans, a major microorganism of human dental caries in the oral cavity, can form biofilms on the teeth [1]. Their aggregation ability allows the colonization of $S$. mutans on the surface of human teeth, as a first step in the development of biofilms [2]. Thereafter, $S$. mutans glucosyltransferases $(g t f)$ begin to synthesize glucan, which increases the adherence of bacteria. In the presence of sucrose, gtfs also produce extracellular polysaccharides (EPSs) from sucrose [3]. Among the EPSs, glucan mediates the initial stage of adherence of oral bacteria on the tooth surface and stimulates the biofilm [4]. Once biofilm forms on a tooth surface, not only is it difficult to remove but also infects other tooth surfaces [5].

Dental caries, caused by S. mutans, is an oral disease affecting the health of adults and children [3]. According to the World Health Organization (WHO) report, more than 530 million children globally have dental caries in their primary teeth [6]. At present, dental caries has been treated using mechanical removal, such as tooth brushing and treatment with mouthwashes containing chlorhexidine and fluoride [7]. However, these methods can also destroy the ecological balance of the oral cavity or damage tissues [8].

Probiotics are live bacteria that can modulate the intestinal microflora when ingested in adequate amounts by the host $[9,10]$. The characteristic properties of probiotics can prevent invasion and cellular adhesion of pathogenic bacteria [11]. In recent decades, the use of probiotics to prevent oral infections has significantly increased. Some of the specific Lactobacillus strains have shown the potential to interfere with oral ecology by inhibiting pathogenic bacteria such as $S$. mutans $[12,13]$. Therefore, this study aims to identify antibacterial and antibiofilm effects of Lactobacillus brevis strains isolated from Korean fermented foods against oral pathogenic S. mutans strains. 


\section{Materials and Methods}

\section{Bacterial Strains and Growth Conditions}

L. brevis strains were isolated using lactobacilli MRS (BD Bioscience, USA). Lactobacillus rhamnosus GG (Cell Biotech, Ltd., Korea) was used as a commercial control strain and obtained from the Korean Collection for Type Cultures (Korea). All Lactobacillus strains were cultured in MRS broth at $37^{\circ} \mathrm{C}$ for $24 \mathrm{~h}$.

S. mutans KCTC 5124, KCTC 5458, and KCTC 5316 were obtained from the Korean Collection for Type Cultures. The strains were cultured in brain heart infusion broth (BHI, BD Bioscience, USA) supplemented with $3 \%$ sucrose at $37^{\circ} \mathrm{C}$ for $24 \mathrm{~h}$ and used as oral pathogenic bacteria.

\section{Preparation Cell-Free Supernatant (CFS) of Lactobacillus Strains}

Lactobacillus strains were cultured in MRS broth at $37^{\circ} \mathrm{C}$ for $24 \mathrm{~h}$. The CFSs of the strains CFS were centrifuged $\left(12,000 \times g\right.$ for $10 \mathrm{~min}$ at $\left.4^{\circ} \mathrm{C}\right)$ and filtered through a $0.45-\mu \mathrm{m}$ pore size syringe filter (Advantec, Japan) (pH 4.3) and stored at $-80^{\circ} \mathrm{C}$ until further use.

\section{Antibacterial Effect of L. brevis Strains against S. mutans Strains}

The antibacterial effect of $L$. brevis strains against $S$. mutans strains was investigated using the deferred method with minor modifications [14]. L. brevis strains $\left(3 \mu \mathrm{l} ; 1 \times 10^{9} \mathrm{CFU} / \mathrm{mL}\right)$ were spotted onto MRS agar and incubated at $37^{\circ} \mathrm{C}$ for $24 \mathrm{~h}$. S. mutans strains $(100 \mu \mathrm{l})$, as an indicator of oral diseases $\left(1 \times 10^{7} \mathrm{CFU} / \mathrm{ml}\right)$, were inoculated into $4 \mathrm{ml}$ of BHI soft agar containing $3 \%$ sucrose, followed by soft agar overlay. The plate was incubated at $37^{\circ} \mathrm{C}$ for $24 \mathrm{~h}$ in an anaerobic incubator. Clear zones were measured and expressed in terms of millimeters.

\section{Minimum Inhibitory Concentration (MIC) of L. brevis Strains against S. mutans Strains}

The MIC of $L$. brevis strains against $S$. mutans strains was investigated using the method described by Lim et al. [4] with some modifications. CFSs of the L. brevis strains were serially diluted two-fold using BHI broth containing $3 \%$ sucrose ranging from $100 \%$ to $0.78 \%$ in a 96 well plate (SPL, Korea). S. mutans strains $\left(1 \times 10^{7} \mathrm{CFU} / \mathrm{ml}\right)$ and diluted CFSs were added to each well. The lowest sample concentration that inhibited $99 \%$ of the inoculum was considered the MIC.

\section{Cell Surface Properties}

The auto-aggregation and cell surface hydrophobicity of S. mutans KCTC 5458 were evaluated using the method of Sorroche et al. [15] with minor modifications. Five milliliters of $S$. mutans KCTC $5458\left(1 \times 10^{7} \mathrm{CFU} / \mathrm{ml}\right)$ was mixed with $5 \mathrm{ml}$ of CFS diluted to $1 / 2 \times$ MIC in BHI broth and incubated at $37^{\circ} \mathrm{C}$ for $24 \mathrm{~h}$ under anaerobic conditions; non-treated cells were used as a control. After incubation, cells were centrifuged $(12,000 \times g$ for $10 \mathrm{~min}$ at $4^{\circ} \mathrm{C}$ ), and the pellet was washed twice and resuspended with phosphate-buffered saline (PBS, pH 7.4; Hycolone, USA) to an optical density (OD) of $0.5 \pm 0.05$ at $600 \mathrm{~nm}\left(\mathrm{OD}_{\text {initial }}\right)$. The suspensions were incubated at $37^{\circ} \mathrm{C}$ for $24 \mathrm{~h}$. Thereafter, the $\mathrm{OD}$ at $600 \mathrm{~nm}$ was measured for suspension $\left(\mathrm{OD}_{\text {Treatment }}\right)$. Auto-aggregation (\%) was calculated as follows:

$$
\text { Auto-aggregation }(\%)=\left(1-\frac{\mathrm{OD}_{\text {Treatment }}}{\mathrm{OD}_{\text {Initial }}}\right) \times 100
$$

After washing the cells, the absorbance at $600 \mathrm{~nm}\left(\mathrm{OD}_{\text {Initial }}\right)$ was adjusted to $0.5 \pm 0$. Chloroform $(0.5 \mathrm{ml})$ was added to each cell suspension $(2 \mathrm{ml})$ and pre-incubated for $10 \mathrm{~min}$ at $37^{\circ} \mathrm{C}$. Thereafter, the mixtures were vortexed for $2 \mathrm{~min}$ and incubated for $15 \mathrm{~min}$ at $37^{\circ} \mathrm{C}$. The aqueous phase was measured at $600 \mathrm{~nm}\left(\mathrm{OD}_{\text {Treatment }}\right)$. The cell surface hydrophobicity was calculated using the following formula:

$$
\text { Cell surface hydrophobicity }(\%)=\left(1-\frac{\mathrm{OD}_{\text {Treatment }}}{\mathrm{OD}_{\text {Initial }}}\right) \times 100
$$

\section{Analysis of Total EPS Production Rate}

EPS production by S. mutans KCTC 5458 was measured by the phenol-sulfuric acid method with some modifications [16]. Five milliliters of S. mutans KCTC 5458 diluted to $10^{7} \mathrm{CFU} / \mathrm{ml}$ in BHI broth containing 3\% sucrose was mixed with $5 \mathrm{ml}$ of CFS diluted to $1 / 2 \times$ MIC in BHI broth containing $3 \%$ sucrose and incubated at $37^{\circ} \mathrm{C}$ for $24 \mathrm{~h}$ under anaerobic conditions; non-treated cells were used as a control. After incubation, the treated mixtures were centrifuged at $12,000 \times g$ at $4^{\circ} \mathrm{C}$ for $10 \mathrm{~min}$, and $1 \mathrm{ml}$ of supernatant was mixed with $2 \mathrm{ml}$ of $99 \%$ ethyl alcohol and incubated for $24 \mathrm{~h}$ at $4^{\circ} \mathrm{C}$. After incubation, the mixture was centrifuged at 14,240 $\times \mathrm{g}$ for $15 \mathrm{~min}$, and the pellets were resuspended in $500 \mu \mathrm{l}$ of distilled water. In the cell suspension $(100 \mu \mathrm{l}), 5 \%$ phenol $(100 \mu \mathrm{l})$, and $95 \%$ sulfuric acid ( $4 \mathrm{ml}$ ) were mixed; the mixture was vortexed, and incubated at $30^{\circ} \mathrm{C}$ for $10 \mathrm{~min}$. The absorbance was calculated using the following formula:

$$
\text { EPS production rate }(\%)=\frac{\mathrm{OD}_{\text {Treatment }}}{\mathrm{OD}_{\text {Control }}} \times 100
$$

\section{Biofilm Assay}

Biofilm inhibition and eradication were measured using a crystal violet assay, with some modifications [17]. Overnight cultured S. mutans KCTC 5458 was diluted to $10^{7} \mathrm{CFU} / \mathrm{ml}$ in BHI broth containing 3\% sucrose. To determine the inhibitory effect of $L$. brevis CFS on the formation of $S$. mutans biofilm, $50 \mu$ of bacterial cultures and $50 \mu \mathrm{l}$ of CFS diluted to $1 / 2 \times$ MIC in BHI broth containing $3 \%$ sucrose were transferred to a 96 well plate and 
incubated at $37^{\circ} \mathrm{C}$ for $24 \mathrm{~h}$; untreated cells were used as a control. After biofilm formation, the cell suspensions were removed using a micropipette. The plates were washed twice with $150 \mu \mathrm{l}$ of PBS. Plates were dried at $37^{\circ} \mathrm{C}$ for $20 \mathrm{~min}$. Thereafter, $1 \%$ crystal violet was added to each well to stain the biofilm-forming cells for $30 \mathrm{~min}$ at room temperature. After dyeing, the plate was rinsed and dissolved in a solution of $30 \%$ methanol and $10 \%$ acetic acid. The OD of each sample was measured at $570 \mathrm{~nm}$ using a microplate reader (Molecular Devices, USA).

To investigate the effect of eradication on the formation of $S$. mutans biofilms, the cell density was adjusted to $10^{7} \mathrm{CFU} / \mathrm{ml}$ in BHI broth containing 3\% sucrose, and $100 \mu \mathrm{l}$ of cell suspension was inoculated into 96 well plate and incubated at $37^{\circ} \mathrm{C}$ for $24 \mathrm{~h}$ under anaerobic conditions; non-treated cells were used as a control. After incubation, each well was washed twice with $150 \mu \mathrm{l}$ PBS. One hundred microliters of $L$. brevis CFS $(1 / 2 \times \mathrm{MIC}$ and MIC) was added to each well and incubated at $37^{\circ} \mathrm{C}$ for $24 \mathrm{~h}$. Non-treated cells were used as a control. The results were quantified as follows.

Biofilm inhibition and eradication rate $(\%)=\left(1-\frac{\mathrm{OD}_{\text {Treatment }}}{\mathrm{OD}_{\text {Initial }}}\right) \times 100$

\section{Scanning Electron Microscopy (SEM) Analysis}

SEM was performed to investigate the biofilm inhibition effect of L. brevis KCCM 202399 CFS on S. mutans KCTC 5458 biofilm using a modified method [18]. Overnight cultured S. mutans KCTC 5458 was diluted to $10^{7} \mathrm{CFU} / \mathrm{ml}$ using BHI broth containing 3\% sucrose. Two milliliters of bacterial suspension and $2 \mathrm{ml}$ of CFS diluted to $1 / 2 \times$ MIC in $\mathrm{BHI}$ broth containing $3 \%$ sucrose were cultured in each well of a six-well plate containing glass coupons and incubated at $37^{\circ} \mathrm{C}$ for $24 \mathrm{~h}$ under anaerobic conditions. The control group was treated with $\mathrm{BHI}$ broth containing $3 \%$ sucrose. The biofilms formed on the glass coupons were fixed with $2.5 \%$ glutaraldehyde in PBS at $4^{\circ} \mathrm{C}$ for $1 \mathrm{~h}$. The fixed samples were washed twice with PBS and dehydrated for 30 min using gradually increasing concentrations of ethanol solutions (50\%, 70\%, 80\%, 90\%, and 100\%). Ethanol was replaced with isoamyl acetate, and the coupons were dried in a freeze dryer and then coated with platinum particles $(15 \mathrm{mV}$ for $1.5 \mathrm{~min}$ ). The S. mutans KCTC 5458 biofilm was observed using a field-emission scanning electron microscope (FESEM; SU8010; Hitachi High-Technologies Co., Japan).

Confocal Laser Scanning Microscopy (CLSM) Analysis

CLSM was performed to evaluate the biofilm inhibition effect of L. brevis KCCM 202399 CFS on S. mutans KCTC 5458 biofilm. Biofilms of S. mutans KCTC 5458 were prepared using the same protocol as described in section 2.8. After biofilm formation, the glass coupons were washed twice with PBS. Live and dead cells were stained with $1 \mu \mathrm{M}$ SYTO9 and propidium iodide (PI) for $20 \mathrm{~min}$ in the dark at room temperature. After staining, the glass coupons were washed twice with PBS and observed under a Zeiss LSM 800 microscope (Carl Zeiss, Germany) using a $10 \times$ objective lens and an appropriate standard filter.

\section{Statistical Analysis}

All experiments were repeated three times with duplicate samples, and the results are presented as the mean \pm standard deviation. All statistical analyses were performed using SPSS 18.0. Significant differences among means were determined using one-way analysis of variance (ANOVA).

\section{Results}

Antibacterial Effect against S. mutans Strains

The antibacterial effects of Lactobacillus strains against $S$. mutans strains are presented in Table 1. Among S. mutans strains, L. brevis strains showed a higher antibacterial effect against $S$. mutans KCTC 5458 than against S. mutans KCTC 5124 and S. mutans KCTC 5316, except for L. rhamnosus GG. Antibacterial effects of L. rhamnosus GG were $7.55 \pm 1.3 \mathrm{~mm}$ and $9.11 \pm 1.0 \mathrm{~mm}$ against $S$. mutans KCTC 5124 and $S$. mutans KCTC 5316, respectively (Table 1; $p<0.05$ ). L. brevis strains showed a high antibacterial effect against $S$. mutans KCTC 5458 (all Lactobacillus strains examined had an inhibition zone over $10 \mathrm{~mm}$ ). L. rhamnosus GG and L. brevis KCCM 202399 showed higher antibacterial effect against $S$. mutans KCTC 5458 than other L. brevis strains $(16.66 \pm 1.0 \mathrm{~mm}$ and

Table 1. Inhibition effect of Lactobacillus strains against Streptococcus mutans strains.

\begin{tabular}{|c|c|c|c|c|c|c|}
\hline \multirow{2}{*}{$\begin{array}{l}\text { Oral pathogenic } \\
\text { bacteria }\end{array}$} & \multicolumn{6}{|c|}{ Inhibitory diameter $(\mathrm{mm})$} \\
\hline & $\mathrm{LGG}^{1)}$ & KCCM 2023992) & KU15159 3 ) & KU15147 ${ }^{4)}$ & KCCM 2023025) & KCCM 2000196) \\
\hline S. mutans KCTC 5124 & $7.55 \pm 1.33^{\mathrm{a}}$ & $7.66 \pm 0.86^{\mathrm{a}}$ & $5.66 \pm 0.86^{\mathrm{b}}$ & $6.33 \pm 1.22^{\mathrm{a}}$ & $6.11 \pm 1.16^{\mathrm{b}}$ & $4.88 \pm 1.05^{\mathrm{b}}$ \\
\hline S. mutans KCTC 5458 & $16.27 \pm 2.10^{\mathrm{a}}$ & $16.11 \pm 1.45^{\mathrm{a}}$ & $13.33 \pm 1.73^{\mathrm{bc}}$ & $14.83 \pm 0.93^{\mathrm{ab}}$ & $11.72 \pm 1.60^{\mathrm{cd}}$ & $10.66 \pm 1.32^{\mathrm{d}}$ \\
\hline S. mutans KCTC 5316 & $9.44 \pm 1.42^{\mathrm{a}}$ & $8.56 \pm 1.67^{\mathrm{a}}$ & $6.89 \pm 0.78^{b}$ & $6.33 \pm 1.00^{\mathrm{b}}$ & $6.78 \pm 2.05^{\mathrm{b}}$ & $5.33 \pm 0.71^{\mathrm{b}}$ \\
\hline
\end{tabular}

${ }^{1-6)}$ LGG, L. rhamnosus GG; KCCM 202399, L. brevis KCCM 202399; KU15159, L. brevis KU15159; KU15147, L. brevis KU15147; KCCM 202302, L. brevis KCCM 202302; KCCM 200019, L. brevis KCCM 200019.

All values are mean \pm standard deviation.

${ }^{\text {a-d }}$ Values with different letters in the same row are significantly different $(p<0.05) .{ }^{1-6)}$ LGG, L. rhamnosus GG; KCCM 202399,

L. brevis KCCM 202399; KU15159, L. brevis KU15159; KU15147, L. brevis KU15147; KCCM 202302, L. brevis KCCM 202302;

KCCM 200019, L. brevis KCCM 200019.

All values are mean \pm standard deviation.

${ }^{\mathrm{a}-\mathrm{d}}$ Values with different letters in the same row are significantly different $(p<0.05)$. 
Table 2. Antibacterial effect of the Lactobacillus strains cell free supernatant (CFS) against S. mutans strains.

\begin{tabular}{ccccccc}
\hline \multirow{2}{*}{$\begin{array}{c}\text { Oral pathogenic } \\
\text { bacteria }\end{array}$} & \multicolumn{5}{c}{ Minimal inhibitory concentration (\%) } \\
\cline { 2 - 6 } & LGG $^{1)}$ & KCCM 202399 $^{2)}$ & KU15159 $^{3)}$ & KU15147 $^{4)}$ & KCCM 202302 $^{5)}$ & KCCM 2000196) $^{\text {a }}$ \\
\hline S. mutans KCTC 5124 & $25 \pm 0.0^{\mathrm{a}}$ & $25 \pm 0.0^{\mathrm{a}}$ & $50 \pm 0.0^{\mathrm{b}}$ & $50 \pm 0.0^{\mathrm{b}}$ & $50 \pm 0.0^{\mathrm{b}}$ & $50 \pm 0.0^{\mathrm{b}}$ \\
S. mutans KCTC 5458 & $6.25 \pm 0.0^{\mathrm{a}}$ & $6.25 \pm 0.0^{\mathrm{a}}$ & $12.5 \pm 0.0^{\mathrm{b}}$ & $12.5 \pm 0.0^{\mathrm{b}}$ & $25 \pm 0.0^{\mathrm{c}}$ & $25 \pm 0.0^{\mathrm{c}}$ \\
S. mutans KCTC 5316 & $25 \pm 0.0^{\mathrm{a}}$ & $25 \pm 0.0^{\mathrm{a}}$ & $50 \pm 0.0^{\mathrm{b}}$ & $50 \pm 0.0^{\mathrm{b}}$ & $50 \pm 0.0^{\mathrm{b}}$ & $50 \pm 0.0^{\mathrm{b}}$ \\
\hline
\end{tabular}

${ }^{1-6)}$ LGG, L. rhamnosus GG; KCCM 202399, L. brevis KCCM 202399; KU15159, L. brevis KU15159; KU15147, L. brevis KU15147; KCCM 202302, L. brevis KCCM 202302; KCCM 200019, L. brevis KCCM 200019.

All values are mean \pm standard deviation.

${ }^{\mathrm{a}-c}$ Values with different letters in the same row are significantly different $(p<0.05)$.

$16.00 \pm 1.0 \mathrm{~mm}$, respectively; Table 1 ). Table 2 shows the MIC of L. brevis strains CFS against S. mutans strains. L. brevis strains CFS showed antibacterial effects against S. mutans KCTC 5458, except for S. mutans KCTC 5124 and S. mutans KCTC 5316. The MICs of L. rhamnosus GG were $25 \%, 6.25 \%$, and $25 \%$ against $S$. mutans KCTC 5124, S. mutans KCTC 5458, and S. mutans KCTC 5316, respectively, while those of L. brevis KCCM 202399 were $25 \%, 6.25 \%$, and 25\%, respectively. L. brevis KCCM 202399 CFS showed better antibacterial effects against S. mutans strains than the other L. brevis strains.

\section{Cell Surface Properties}

The effects of $L$. brevis strains CFS on auto-aggregation and cell surface hydrophobicity of S. mutans KCTC 5458 are shown in Table 3. Treatment with L. brevis strains CFS decreased auto-aggregation $(p<0.05)$ and cell-surface hydrophobicity $(p<0.05)$ of $S$. mutans KCTC 5458, compared with the negative control. The auto-aggregation ability of S. mutans KCTC 5458 treated with L. brevis KCCM 202399 and L. brevis KU15147 decreased by $45.91 \%$ and $49.11 \%$, respectively. Additionally, S. mutans KCTC 5458 treated with L. rhamnosus GG was $46.35 \%$. The cell surface hydrophobicity of S. mutans KCTC 5458 treated with L. rhamnosus GG CFS and L. brevis KCCM 202399 CFS was $32.97 \%$ and $40.51 \%$, respectively. Our results showed that Lactobacillus strains CFS inhibited bacterial adhesion by decreasing the auto-aggregation and cell-surface hydrophobicity of S. mutans KCTC 5458.

\section{EPS Production Rate}

The EPS production rate of S. mutans KCTC 5458 treated with L. brevis strains CFS was evaluated using a modified phenol-sulfuric acid method [16]. Fig. 1 presents the inhibitory effect of L. brevis strain CFS on EPS production rate $(p<0.05)$. Treatment with L. brevis KCCM 202399 CFS resulted in the lowest EPS production rate (67.44\%). For treatment with $L$. rhamnosus GG CFS, the EPS production rate was $72.35 \%$, followed by treatment with L. brevis KU15147, and L. brevis KCCM 200019 (73.66\% and 76.44\%, respectively). As shown in Fig. 1 and Supplementary Tables 1, 2, and 3, L. brevis KCCM 202399, L. brevis KU15159, and L. brevis KU15147 CFS had a greater inhibitory effect on $S$. mutans growth and EPS production than other L. brevis strains.

\section{Biofilm Inhibition and Eradication Effects of CFS}

The inhibitory effect of L. brevis CFS on S. mutans KCTC 5458 biofilm is shown in Fig. 2A $(p<0.05)$; the concentration of CFS ranging from $1 / 2 \times$ MIC and MIC inhibited the formation of S. mutans biofilm. Upon treatment with CFS at MIC concentration, L. brevis KCCM 202399 showed highest inhibition effects against S. mutans (68.54\%). In addition, the inhibition effect of L. rhamnosus GG CFS was $70.05 \%$. This inhibitory tendency was similarly observed at $1 / 2$ MIC concentrations. Upon treatment with CFS at $1 / 2 \mathrm{MIC}$ concentrations, the inhibition effects of L. rhamnosus GG CFS and L. brevis KCCM 202399 CFS were $41.45 \%$ and $35.28 \%$, respectively. The degradation effect of $L$. brevis CFS on mature biofilms is shown in Fig. 2B $(p<0.05)$. Upon treatment with CFS MIC, the degradation effect of L. rhamnosus GG CFS, L. brevis KCCM 202399 CFS, and L. brevis KU15147 CFS was 51.19\%, 50.29\%, and 45.44\%, respectively. However, upon treatment with CFS at $1 / 2$

Table 3. Effects of the cell-free supernatant of Lactobacillus strains on auto-aggregation and cell surface hydrophobicity of S.mutans KCTC 5458.

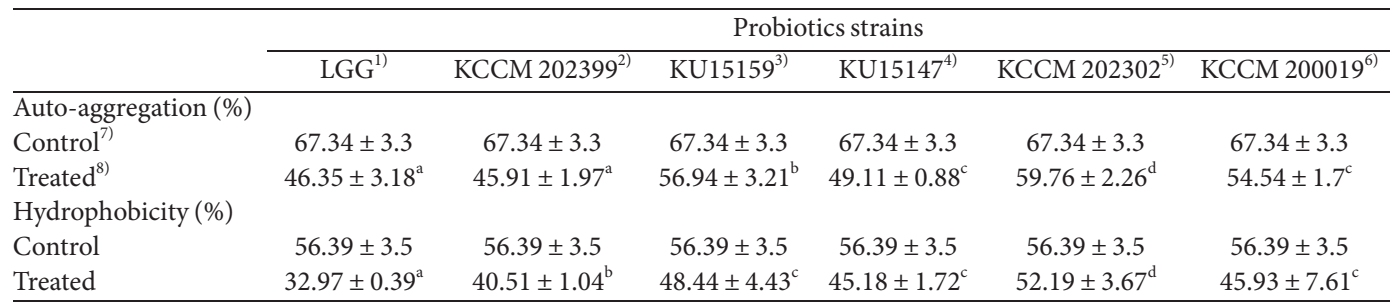

${ }^{1-8)}$ LGG, L. rhamnosus GG; KCCM 202399, L. brevis KCCM 202399; KU15159, L. brevis KU15159; KU15147, L. brevis KU15147; KCCM 202302, L. brevis KCCM 202302; KCCM 200019, L. brevis KCCM 200019; control, treated with probiotic CFS.

${ }^{a-c}$ Values with different letters in the same row are significantly different $(\mathrm{p}<0.05)$.

All values are mean \pm standard deviation. 


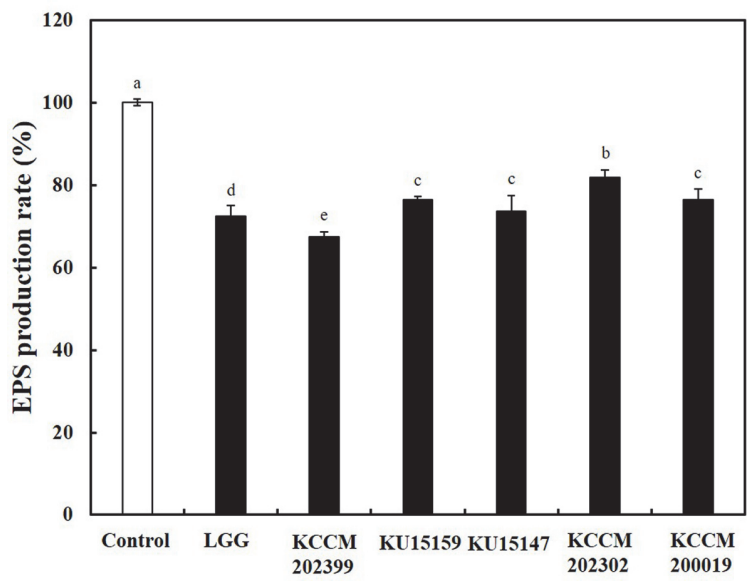

Fig. 1. EPS production rate of Streptococcus mutans KCTC 5458 treated with cell-free supernatant (CFS) of Lactobacillus brevis strains. $\square$, Control (treated L. brevis strains); $\square$, L. brevis strains; LGG, L. rhamnosus GG. Each value represents the mean \pm standard deviation, with ${ }^{\text {a-d }}$ different letters on each bar representing significant differences $(p<0.05)$.

MIC concentrations, L. brevis KCCM 202399 CFS showed a greater degradation effect than the other L. brevis strains CFS $(39.75 \%$; $p<0.05)$. The biofilm inhibition and degradation effects of L. brevis CFS were dosedependent. As shown in Fig. 2, L. brevis KCCM 202399 CFS showed greater biofilm inhibition and eradication effects against $S$. mutans than other L. brevis strains CFS $(p<0.05)$.

\section{SEM Analysis on Glass Coupon}

The effects of L. brevis KCCM 202399 CFS on biofilm formation by S. mutans KCTC 5458 on glass coupons were also evaluated by SEM (Fig. 3). In the control group, S. mutans KCTC 5458 formed numerous bacterial cells and a large biofilm on the glass coupons (Fig. 3A). However, biofilm structures of S. mutans KCTC 5458 treatedL. rhamnosus GG CFS and L. brevis KCCM 202399 CFS were spread, resulting in decreased bacterial cells and biofilms on glass coupons (Figs. 3B and 3C). Considering the results of the other experiments, including cell surface properties (Table 3), EPS production (Fig. 1), biofilm inhibition and degradation effects (Fig. 2), the inhibitory effect of probiotic L. brevis KCCM 202399 CFS from S. mutans biofilm was confirmed.

\section{CLSM Analysis on Glass Coupon}

The antibiofilm and antibacterial effects of L. brevis KCCM 202399 CFS against S. mutans KCTC 5458 on glass coupons were observed via CLSM. CLSM images showed S. mutans biofilms with viable and non-viable cells (green and red, respectively). In the control, S. mutans KCTC 5458 showed a dense biofilm structure and biofilm cells (Fig. 4A1, 2). However, the biofilm structures of L. rhamnosus GG CFS- and L. brevis KCCM 202399 CFStreated S. mutans KCTC 5458 were decreased. Furthermore, L. brevis KCCM 202399 CFS showed significantly reduced viability of $S$. mutans KCTC 5458 biofilm cells compared with L. rhamnosus GG CFS (Figs. 4B and 4C).
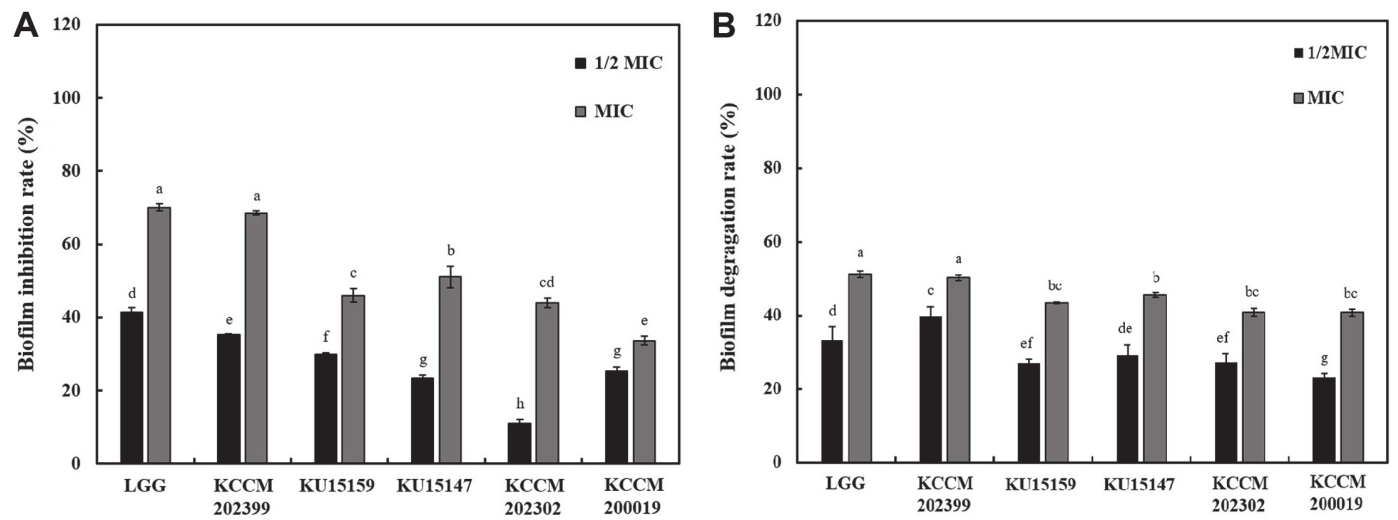

Fig. 2. Antibiofilm effects of cell-free supernatant (CFS) of Lactobacillus brevis strains on Streptococcus mutans KCTC 5458. (A), Biofilm inhibition effect of CFS; (B), Biofilm eradication effect of CFS. LGG, L. rhamnosus GG; KCCM 202399, L. brevis KCCM 202399; KU15159, L. brevis KU15159; KU15147, L. brevis KU15147; KCCM 202302, L. brevis KCCM 202302; KCCM 200019, L. brevis KCCM 200019. Each value represents the mean \pm standard deviation, with ${ }^{\text {a-g }}$ different letters on each bar representing significant differences $(p<0.05)$. 

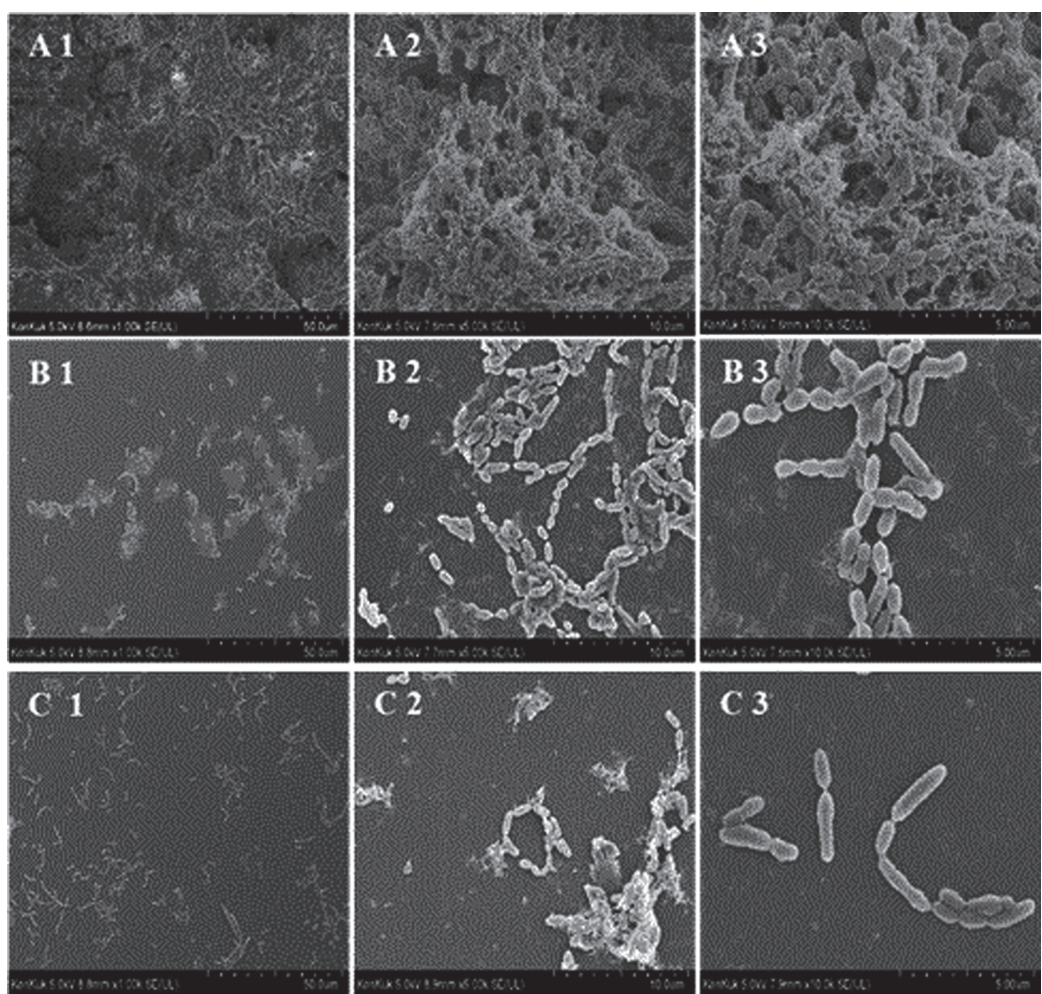

Fig. 3. Streptococcus mutans KCTC 5458 biofilm on glass coupons surface treatment with cell-free supernatant (CFS) of Lactobacillus brevis KCCM 202399 visualized by scanning electron microscopy (SEM) images (magnification: $\times \mathbf{1 , 0 0 0} \times \mathbf{5}, \mathbf{0 0 0}$, and $\times \mathbf{1 0 , 0 0 0}$ ). A group: control group (untreated with L. brevis CFS); $\mathrm{B}$ group: treated with $L$. rhamnosus GG CFS; C group: treated with L. brevis KCCM 202399 CFS.
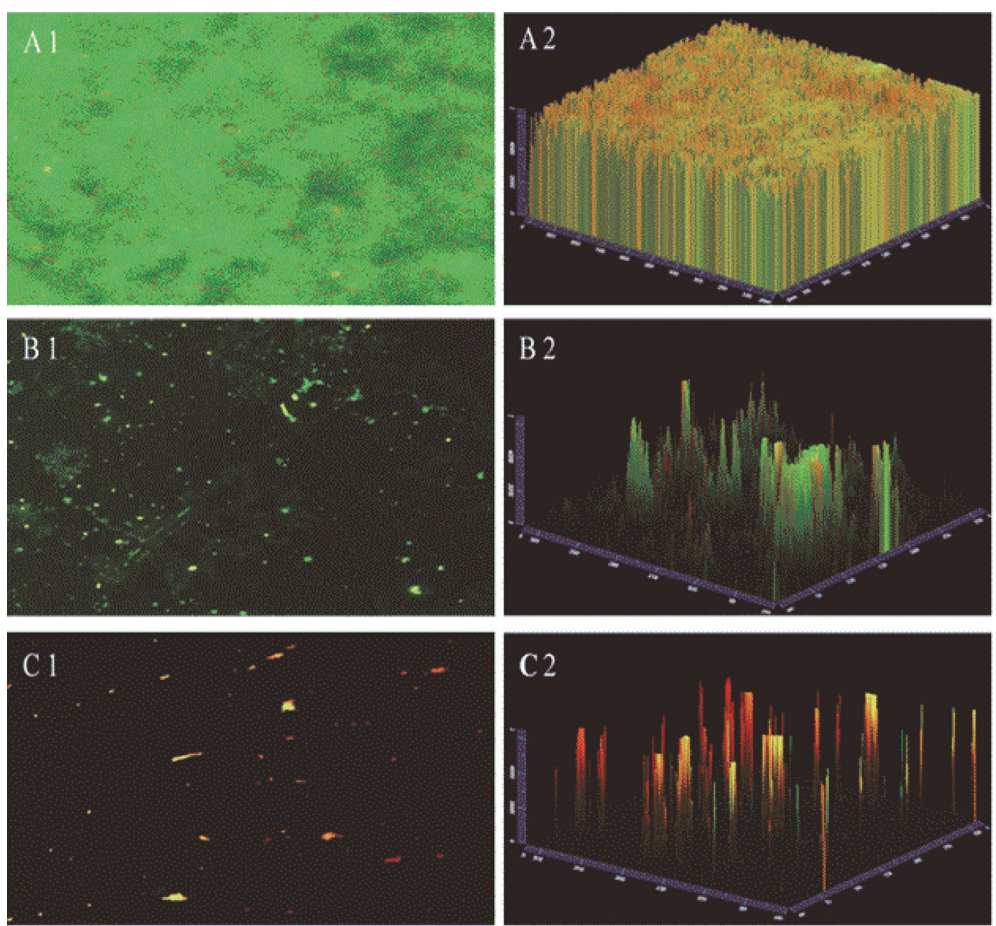

Fig. 4. Confocal laser scanning microscopy (CLSM) images of Streptococcus mutans KCTC 5458 biofilm on glass coupons surface treated with Lactobacillus brevis KCCM 202399 cell-free supernatant (CFS) $(\times 100$ magnification). A group: control group (untreated with L. brevis CFS); B group: treated with L. rhamnosus GG CFS; C group: treated with L. brevis KCCM 202399 CFS. 


\section{Discussion}

Dental caries is a major oral disease that is multi-species biofilm-mediated. Dental plaque, which is a multispecies biofilm, is transformed from cariogenic to non-cariogenic plaque. S. mutans is a cariogenic bacteria in dental plaque that colonizes the tooth surface and forms biofilms [5]. Once S. mutans forms a biofilm, it is difficult to remove; therefore, its early control is important. This study was aimed at investigating the antibacterial and antibiofilm effects of L. brevis strains isolated from kimchi.

In this study, methods were developed to screen the antibacterial effect of $L$. brevis strains against $S$. mutans strains. The results showed that L. brevis strains showed a higher antibacterial effect against S. mutans KCTC 5458 than against S. mutans KCTC 5124 and S. mutans KCTC 5316. Thereafter, antibacterial effect of L. brevis strains against $S$. mutans strains was investigated by using L. brevis CFS; it was confirmed that L. brevis strains have a higher antibacterial effect on S. mutans KCTC 5458 than on S. mutans KCTC 5124 and S. mutans KCTC 5316. Some Lactobacilli strains can metabolize sucrose, co-aggregate with $S$. mutans, and are often tolerant to fluoride [19]. Therefore, probiotic supernatant could be safely used as an antibacterial agent for treating dental plaque. Taku et al. [20] reported that the "expression" or "sensitivity" of $g t f$ gene, which synthesizes water in-soluble or soluble glucans from sucrose, would be affected differently in different S. mutans strains. CFSs of all L. brevis strains showed greater antibacterial effect against S. mutans KCTC 5458 than against S. mutans KCTC 5124 and S. mutans KCTC 5316. In particular, L. brevis KCCM 202399 CFS showed an antibacterial effect at the lowest concentration among the $L$. brevis strains (Table 2). Therefore, we focused on the antibacterial effect of $L$. brevis CFS against $S$. mutans KCTC 5458.

Auto-aggregation, cell surface hydrophobicity, and EPS production changes in $S$. mutans are important to prevent $S$. mutans adhesion, colonization, and early biofilm formation [21]. Auto-aggregation of $S$. mutans is beneficial for its adhesion to tooth surface, as the resulting biofilm formed prevents this bacterium from an adverse external environment [22]. S. mutans has a high overall proportion of hydrophobic bacteria, and its cell surface hydrophobicity may play a role in the adherence of oral bacteria to the tooth surface [23]. Therefore, we investigated the effect of $L$. brevis CFS on the auto-aggregation ability and cell surface physiochemical properties of S. mutans KCTC 5458. In this study, L. brevis KCCM 202399 CFS showed the highest reduction in autoaggregation and cell surface hydrophobicity of S. mutans KCTC 5458. In another study, auto-aggregation of S. mutans ATCC 25175 treated with L. brevis BBE-Y52 was higher than that in the presence of other Lactobacillus strains [24]. In addition, Bacillus velezensis K68-treated $L$. brevis strains exhibited increased cell surface hydrophobicity, compared with the untreated control [25]. EPS produced by $S$. mutans is a major factor in biofilm formation. As sucrose exists in oral conditions, $g t f s$ from $S$. mutans plays critical roles in the development of virulent dental plaque [26]. In the presence of $L$. brevis CFS, EPS production by S. mutans KCTC 5458 decreased. In particular, L. brevis KCCM 202399 CFS showed the highest reduction in EPS production by S. mutans. These results suggest that $L$. brevis KCCM 202399 reduces sucrose-dependent EPS production by downregulating $g t f$. In our previous study, L. brevis KU15153 CFS decreased EPS production by approximately $41 \%(p<0.05)$ [27]. A previous study reported that biosurfactants produced by probiotics have antibacterial and anti-adhesive properties [28]. The biosurfactants in metabolites exuded by Lactobacilli interfere with the adhesion of cells. These decrease the hydrophobicity of the cell surface substratum and interfere with the progression of microbial adhesion ability [29]. In addition, Tahmourespour et al. [30] reported that Lactobacillus acidophilus-derived biosurfactant down-regulated $g t f s \mathrm{~B}$ and $\mathrm{C}$ genes, and virulence factors were associated with glucan in dental plaque.

Changes in cell surface properties and EPS production by L. brevis KCCM 202399 CFS also affected biofilm formation by $S$. mutans KCTC 5458. In this study, 1/2 MIC and MIC concentrations of $L$. brevis CFS were used for treatment and the inhibition rate of $S$. mutans biofilm as a function of CFS concentration was investigated. The biofilm formed by $S$. mutans in the presence of L. brevis strains CFS exhibited a dose-dependent reduction in biomass compared to the control group that did not receive CFS. In particular, L. brevis KCCM 202399 CFS showed the highest inhibitory effect against $S$. mutans KCTC 5458 at 1/2 MIC and MIC levels. In our previous study, L. rhamnosus GG was reported to have a significant antibacterial effect against S. mutans [4]. Ahn et al. [31] reported that lipoteichoic acid of probiotics could inhibit biofilm formation by S. mutans. Additionally, bacteriocin, an antibacterial substance produced by probiotic Lactobacilli, can kill gram-positive bacteria by disrupting their cell membranes, inhibit their growth by lowering $\mathrm{pH}$ and hamper bacterial DNA synthesis by producing hydrogen peroxide [32]. We also conducted SEM and CLSM analyses to investigate the reduction in biofilm formation and viability of biofilm cells as imaging. S. mutans KCTC 5458 treated with L. brevis KCCM 202399 CFS showed that the biofilm was dispersed with little aggregation, and the number of cells on glass coupons was decreased compared with that for the control (Fig. 3). Compared with L. rhamnosus GG, L. brevis KCCM 202399 showed a higher number of dead biofilm cells, as evidenced by PI staining (Fig. 4). Generally, mature biofilms are more difficult to remove than early biofilms. Biofilms can protect oral bacteria, and $S$. mutans can induce membrane proteins to migrate and overcome cellular damage caused by environmental stress [5, 33]. The biofilm degradation effect is also important to examine the antibiofilm effect against S. mutans; however, eradication effect of probiotics on mature biofilms have rarely been reported. L. brevis KCCM 202399 CFS showed the highest biofilm eradication effect against S. mutans KCTC 5458 at 1/2 MIC and MIC levels, demonstrating its action through biofilm formation and disruption of mature biofilm.

Six L. brevis strains isolated from kimchi were tested for antibacterial effects against $S$. mutans strains. The results showed that L. brevis KCCM 202399 had the highest antibacterial and antibiofilm effects among the L. brevis strains. Furthermore, L. brevis KCCM 202399 showed more antibacterial effects against S. mutans KCTC 5458 than against S. mutans KCTC 5124 and S. mutans KCTC 5316. L. brevis KCCM 202399 CFS inhibited and eradicated the biofilm of $S$. mutans KCTC 5458 by decreasing its auto-aggregation, cell-surface hydrophobicity, 
and EPS production. The antibiofilm effects of CFS against S. mutans KCTC 5458 were also confirmed by SEM and CLSM. Therefore, this study suggests that L. brevis KCCM 202399 could be used as a functional food in the food industry.

\section{Conflict of Interests}

The authors have no financial conflicts of interest to declare.

\section{References}

1. Miki M, Hitoshi K. 2010. Role of two-component system of Streptococcus mutans in the adaptive response to the oral environment. J. Oral Biosci. 52: 252-259.

2. Kim HJ, Lee JH. 2020. Anti-biofilm effect of egg yolk phosvitin by inhibition of biomass production and adherence activity against Streptococcus mutans. Food Sci. Anim. Resour. 40: 1001-1013.

3. Salli KM, Forssten SD, Lahtinen SJ. Ouwehand AC. 2016. Influence of sucrose and xylitol on an early Streptococcus mutans biofilm in a dental simulator. Arch. Oral Biol. 70: 39-46.

4. Lim SM, Lee NK, Paik HD. 2020. Antibacterial and anticavity activity of probiotic Lactobacillus plantarum 200661 isolated from fermented foods against Streptococcus mutans. LWT - Food Sci. Technol. 118: 108840.

5. Yimeng C, Huiwei Y, Wujun W, Pengfei P, Yin W, Xinyu W, et al. 2020. Killing Streptococcus mutans in mature bioflm with a combination of antimicrobial and antibioflm peptides. Amino Acids 52: 1-14.

6. World Health Organization. 2003. Oral health to use from https://www.who.int/news-room/fact-sheets/detail/oral-health. Accessed Mar. 25, 2020.

7. Baker JL, Faustoferri RC, Quivey Jr RG. 2017. Acid-adaptive mechanisms of Streptococcus mutans-the more we know, the more we don't. Mol. Oral Microbiol. 32: 107-117.

8. Kim AR, Ahn KB, Yun CH, Park OJ, Perinpanayagam H, Yoo YJ, et al. 2019. Lactobacillus plantarum lipoteichoic acid inhibits oral multispecies biofilm. J. Endod. 45: 310-315.

9. Cai JN, Kim MA, Jung JE, Pandit S, Song KY, Jeon JG. 2015. Effects of combined oleic acid and fluoride at sub-MIC levels on EPS formation and viability of Streptococcus mutans UA159 biofilms. Biofouling 31: 555-563.

10. Lee NK, Kim SY, Han KJ, Eom SJ, Paik HD. 2014. Probiotic potential of Lactobacillus strains with anti-allergic effects from kimchi for yogurt starters. LWT - Food Sci. Technol. 58: 130-134.

11. Tareb R, Bemardeau M, Gueguen M, Vernoux JP. 2013. In vitro characterization of aggregation and adhesion properties of viable and heat-killed forms of two probiotic Lactobacillus strains and interaction with foodborne zoonotic bacteria. especially Campylobacter jejuni. J. Med. Microbiol. 62: 637-649.

12. Cruz AGD, Ranadheera CS, Nazzaro F, Mortazavian A. 2021. Probiotics and Prebiotics in Foods, pp. 59-80. In Michel RM, Pedro HFS, Luciana PM, Segio S, Arthur CO, Flavia F. Chapter 4 - Probiotics and Prebiotic in Oral Health. Academic Press, Massachusetts.

13. Parul C, Renuka D, Anuradha S, Neeru B, \& Mahesh SD. 2020. A critical appraisal of the effects of probiotics on oral health. J. Funct. Foods 70: 103985.

14. Jang HJ, Lee NK, Paik HD. 2019. Probiotic characterization of Lactobacillus brevis KU15153 showing antimicrobial and antioxidant effect isolated from kimchi. Food Sci. Biotechnol. 28: 1521-1528.

15. Sorroche F, Bogino P, Russo DM, Zorreguieta A, Nievas F, Morales GM, et al. 2018. Cell autoaggregation biofilm formation, and plant attachment in a Sinorhizobium meliloti lpsB mutant. Mol. Plant Microbe Interact. 31: 1075-1082.

16. Chiba A, Sugimoto S, Sato F, Hori S, Mizunoe Y. 2015. A refined technique for extraction of extracellular matrices from bacterial biofilms and its applicability. Microb. Biotechnol. 8: 392-403.

17. Song YJ, Yu HH, Kim YJ, Lee NK, Paik HD. 2019. Anti-biofilm activity of grapefruit seed extract against Staphylococcus aureus and Escherichia coli. J. Mircobiol. Biotechnol. 29: 1177-1183.

18. Yu HH, Song YJ, Yu HS, Lee NK, Paik HD. 2020. Investigating the antimicrobial and antibiofilm effects of cinnamaldehyde against Campylobacter spp. using cell surface characteristics. J. Food Sci. 85: 157-164.

19. Badet C, Richard B, Castaing-Debat M, De Flaujac, PM, Dorignac G. 2004. Adaptation of salivary Lactobacillus strains to xylitol. Arch. Oral Biol. 49: 161-164.

20. Taku F, Yutaka T, Tomonori H, Shigetada K, Takashi O, Shizuo S, et al. 1998. Molecular analyses of glucosyltransferase genes among strains of Streptococcus mutans. FEMS Microbiol. Lett. 161: 331-336.

21. Zhang G, Lu M, Liu R, Tian Y, Vu VH, Li Y, et al. 2020. Inhibition of Streptococcus mutans biofilm formation and virulence by Lactobacillus plantarum K41 isolated from traditional sichuan pickles. Front. Microbiol. 11: 774 .

22. Zhang Z, Lyu X, Xu Q, Li C, Lu M, Gong T, et al. 2020. Utilization of the extract of Cedrus deodara (Roxb. Ex D.Don) G. Don against the biofilm formation and the expression of virulence genes of cariogenic bacterium Streptococcus mutans. J. Ethnopharmacol. 257: 112856.

23. Tahmourespour A, Karsa Kermanshahi R, Salehi R, Nabinezhad AAR. 2008. The relationship between cell surface hydrophobicity and antibiotic resistance of Streptococcal strains isolated from dental plaque and caries. Iran. J. Basic Med. Sci. 10: 251-255.

24. Fang F, Xu J, Li Q, Xia X, Du G. 2018. Characterization of a Lactobaciilus brevis strain with potential oral probiotic properties. BMC Microbiol. 18: 221.

25. Yoo Y, Seo DH, Lee H, Cho ES, Song NE, Nam TG, et al. 2019. Inhibitory effect of Bacillus velezensis on biofilm formation by Streptococcus mutans. J. Biotechnol. 298: 57-63.

26. Sun Y, Jiang W, Zhang M, Zhang L, Shen Y, Huang S, et al. 2021. The inhibitory effects of ficin on Streptococcus mutans biofilm formation. Biomed Res. Int. 2021: 6692328.

27. Jang HJ, Kim JH, Lee NK, Paik HD. 2021. Inhibitory effects of Lactobacillus brevis KU15153 against Streptococcus mutans KCTC 5316 causing dental caries. Microb. Pathog. 157: 104938.

28. Sambanthamoorthy K, Feng X, Patel R, Patel S, Paranavitana C. 2014. Antimicrobial and antibiofilm potential of biosurfactants isolated from lactobacilli against multi-drug-resistant pathogens. BMC Microbiol. 14: 197.

29. Jamwal A, Sharma K, Chauhan R, Bansal S, Goel G. 2019. Evaluation of commercial probiotic lactic cultures against biofilm formation by Cronobacter sakazakii. Intest. Res. 17: 192-201.

30. Tahmourespour A, Salehi R, Kermanshahi RK. 2011. Lactobacillus acidophilus-derived biosurfactant effect on GTFB and GTFC expression level in Streptococcus mutans biofilm cells. Braz. J. Microbiol. 42: 330-339.

31. Ahn KB, Baik JE, Park OJ, Yun CH, Han SH. 2018. Lactobacillus plantarum lipoteichoic acid inhibits biofilm formation of Streptococcus mutans. PLoS One 13: e0192694.

32. Lin X, Chen X, Tu W, Wang S, Chen H. 2017. Effect of probiotic Lactobacilli on the growth of Streptococcus mutans and multispecies biofilms isolated from children with active caries. Med. Sci. Monit. 23: 4175-4181.

33. Jeffrey AB. 2004. Virulence properties of Streptococcus. Front. Biosci. 9: 1264-1277. 